\title{
The Glycoprotein Growth Factor Progranulin Promotes Carcinogenesis and has Potential Value in Anti-cancer Therapy
}

Yonghua Zhang and Andrew Bateman*

Endocrine Research Laboratory, Royal Victoria Hospital, McGill University Health Center, Canada

\begin{abstract}
Progranulin (PGRN) is a secreted glycoprotein growth factor with tumorigenic roles in a variety of tumors including, among others, breast, ovarian, prostate, bladder, and liver cancer. In some patients, for example with breast, ovarian or liver cancers, high PGRN expression in tumors correlated with a worse outcome. Studies using cell lines and animal models provide evidence that PGRN promotes tumor cell proliferation, migration and survival, and induces drug resistance. Increasing or decreasing PGRN production enhances or inhibits respectively the growth of PGRN-sensitive tumors in vivo. PGRN activity is associated with p44/42 mitogen-activated protein kinase as well as phosphatidylinositol 3-kinases signaling pathways. In addition, PGRN may stimulate the formation of the tumor stroma. As an extracellular regulator of tumorgenesis, PGRN is a potential therapeutic target and biomarker of prognosis in the treatment of various cancers.
\end{abstract}

\section{Introduction}

Growth factors, their receptors and downstream signaling proteins play a cardinal role in carcinogenesis [1]. In addition, many cancers depend upon hormones such as estrogen or androgens to support their growth. The importance of these extracellular signaling systems as targets in the development of anti-cancer drugs has long been recognized. Despite this progress, cancer continues to be one of the major causes of morbidity and mortality and many types of cancer remain plagued by a high loss of life [2]. The search for additional biological targets to expand the arsenal of potential anti-cancer weapons remains a priority. Here we will explore the hypothesis that progranulin (PGRN) is an extracellular regulator of tumor progression that presents novel opportunities as a therapeutic and prognostic target in the treatment of a variety of different cancers. The evidence will be reviewed (i): that PGRN is often over-produced in cancerous tissue and that this over-production correlates with disease progression, (ii): that experimentally increasing or decreasing the production or bioavailability of PGRN by cancer cells makes them respectively more or less tumorigenic in vivo, (iii): that PGRN stimulates mitosis, blocks apoptosis, including apoptosis due to anti-cancer drugs, and promotes invasion and that this occurs through signaling pathways that are well known to have oncogenic potential, (iv): that PGRN may have additional tumor promoting roles, for example by eliciting tumor stroma production.

Progranulin [3] is also known as Granulin-Epithelin Precursor [4], Proepithelin [5], PC-cell-derived growth factor (PCDGF) [6], Acrogranin [7] and Glycoprotein, 88kDa (GP88). Edman sequencing of a protein called epithelial transforming growth factor (TGFe) revealed a sequence almost identical to an internal portion of PGRN, suggesting that TGFe may be a biologically active fragment of PGRN [8]. PGRN is a secretable glycoprotein [9] consisting of tandem repeats of a 12 cysteine module called the granulin or epithelin domain [10$12,5,7]$. The individual granulin/epithelin modules can be isolated from tissue extracts and urine as individual peptides of approximately $6 \mathrm{kDa}$ $[10,11,13]$, but whether these are biologically active in their own right or simple breakdown products is not fully resolved (See Figure 1). In this article the term PGRN (progranulin) will be used to mean the full length protein and is synonymous with Granulin/Epithelin Precursor, Proepithelin, PC-Cell-derived growth factor, acrogranin and epithelial
Transforming Growth Factor. The $6 \mathrm{kDa}$ peptidic fragments that correspond to individual granulin/epithelin modules will be called grn/ epi peptides. Following NCBI usage the gene will be referred to as GRN.

PGRN is a multifunctional protein that has been implicated in early embryonic development [14,15], bone development [16], inflammation [17-19] and wound repair [20]. Mutational loss of a single copy of the human GRN gene results in a form of early onset dementia, called frontotemporal lobar dementia that is characterized by neuronal atrophy in the frontal and temporal cerebral lobes [21,22]. Many of these proposed biological roles for PGRN are characterized by cell proliferation, improved survival in the face of an apoptotic challenge, and migration through an extracellular matrix. As will be discussed below it is through these activities that PGRN is thought to promote cancer growth and development.

\section{PGRN expression in tumors from different anatomical sites}

PGRN is expressed at higher levels than normal in a number of cancers of different types including carcinomas, sarcomas, gliomas and myelomas. The diversity of anatomical sites in which PGRN has been associated with cancer is suggestive of a significant role for PGRN in tumor biology. Although hard and fast rules cannot be applied, there is a trend, as reviewed below, for PGRN production to be greatest in more invasive stages of cancer progression. In some cases PGRN levels correlate well with clinical parameters such as overall survival and

*Corresponding author: Andrew Bateman, Professor, Department of Medicine McGill University, Endocrine Research Laboratory, Royal Victoria Hospital, 687 Pine Avenue West, Room H5.21, Montreal, Quebec, Canada H3A 1A1, Tel: (514) 934-1934 ext. 35833; E-mail: andrew.bateman@muhc.mcgill.ca

Received August 16, 2011; Accepted October 27, 2011; Published October 29 2011

Citation: Zhang Y, Bateman A (2011) The Glycoprotein Growth Factor Progranulin Promotes Carcinogenesis and has Potential Value in Anti-cancer Therapy. J Carcinogene Mutagene S2:001. doi:10.4172/2157-2518.S2-001

Copyright: @ 2011 Zhang Y, et al. This is an open-access article distributed unde the terms of the Creative Commons Attribution License, which permits unrestricted use, distribution, and reproduction in any medium, provided the original author and source are credited. 
Progranulin is an extracellular protein with $7 \frac{1}{2}$ repeats of a 12 cysteine granulin/epithelin module (GEMs A to G)
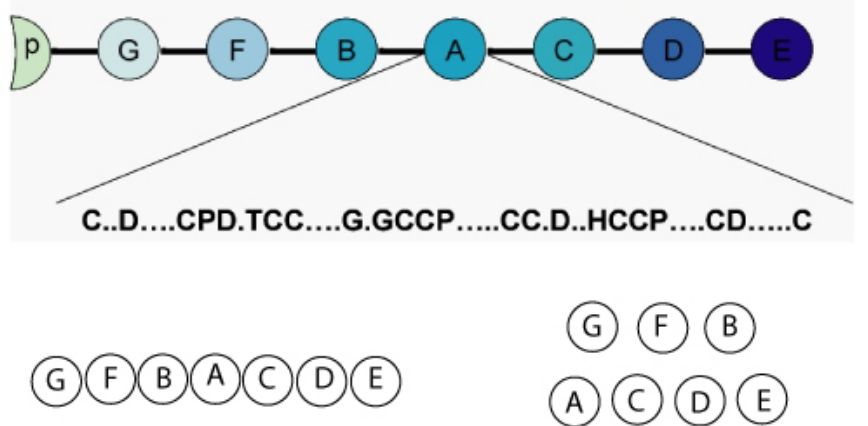

\section{PGRN

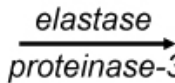

\section{GRN/EPI PEPTIDES}

Figure 1: The structure of Progranulin: The upper panel shows that progranulin is a glycoprotein that is composed of seven and a half repeats of the granulin/ epithelin module designated $A$ to $G$. $P$ represents paragranulin, a six cysteine half module. The conserved amino acids of the granulin/epithelin module are shown (where $C$ is cysteine, $G$ is glycine, $T$ is threonine, $D$ is aspartic acid, $P$ is proline and $\mathrm{H}$ is histidine. Dots represent amino acid residues that are not highly conserved). The lower panel shows that progranulin can be converted to peptide fragments each of about $6 \mathrm{kDa}$ that correspond to the granulin/epithelin modules.

\section{progression free survival.}

Breast cancer: Immunohistochemical analysis of paraffinembedded human breast cancer samples demonstrated that PGRN was overexpressed in $80 \%$ [23] and 79\% [24] of invasive ductal carcinoma and half of invasive lobular carcinoma, whereas almost no detectable PGRN expression was observed in normal mammary epithelium and benign tumors. PGRN expression significantly correlated with the histological grade of invasive and ductal carcinomas in situ [23], and with the Ki-67 index of proliferation in all invasive carcinomas [23]. P53 was more commonly expressed in those invasive carcinomas with high PGRN expression $[23,24]$. There was no significant correlation between PGRN expression and the human epidermal growth factor receptor (HER) family member HER-2. PGRN expression was independent of estrogen and progesterone receptors (ER/PR) status in one study [23], whereas Song et al. [24] reported that PGRN positive staining was more common in ER/PR negative samples than that in ER/PR positive tumors. Elkabets et al. [25] reported that high level PGRN staining was significantly associated with breast tumor size, histological and molecular subtypes. High PGRN expression correlated negatively with the luminal A subtype which are generally low grade tumors that express ER [26], but was positively correlated with triple negative breast cancer (tumors with negative expression of ER, PR, and HER-2 [27]) and basal-like breast cancer subtypes [25]. These cancers are often difficult to treat, suggesting that a PGRN-based therapy could significantly supplement existing breast cancer treatment. PGRNpositive expression in cancer specimens correlated with significantly worse overall survival compared to those without PGRN-staining [25]. Recent work demonstrates that PGRN levels are elevated in the serum of patients with breast cancer [28]. In contrast to these studies however, comparing gene expression profiles between 10 tumors from node-positive patients who survived for 5 years after surgery and 10 tumors from those patients who died of breast cancer within 5 years, Asaka et al. [29] concluded that PGRN was significantly downregulated among the patients who died or show worse outcomes and proposed that downregulated expression of PGRN is a prognostic indicator for subdividing node-positive patients into finer groups with "good prognosis".

Ovarian cancer: Ovarian cancer is the deadliest gynecologic malignancy and around $60 \%$ of women with it will die from the cancer [30]. In one immunohistochemistry analysis on human primary ovarian carcinoma, effusions, and tissues of metastatic lesions, PGRN protein expression was observed in $95 \%$ of ovarian solid tumor specimens, and the expression was detected in all tissue compartments of the ovarian carcinoma, including carcinoma cells, reactive stromal cells, and tumor-associated endothelial cells [31]. In a statistical analysis of GRN gene expression in laser microdissected ovarian tumor epithelium, GRN expression was observed only in invasive ovarian cancer epithelium and was absent in tumors with low malignant potential (LMP) [32]. Among LMP lesions, PGRN protein expression was, however, occasionally detected in the stromal cells. Miyanishi et al. [33] also reported that immunohistochemical staining of PGRN in the epithelial lesion of ovarian cancer is significantly stronger than that in LMP. PGRN has been investigated as a potential prognostic marker for ovarian cancer. A significantly worse overall survival for patients with high PGRN mRNA expression in ovarian cancers was demonstrated [34]. High PGRN protein expression in ovarian effusion tumor cells correlated to a better overall survival, while elevated PGRN expression in the tumor stromal cells correlated with worse overall survival [31]. Han and et al. [35] studied the relationship between the expression of several serum prediction biomarkers for epithelial ovarian cancer among patients in complete clinical remission. Using receiver operating characteristic and area under the curve analyses to define optimal cut-off points, PGRN levels were significantly associated with worse progression-free survival and overall survival. Elevated PGRN levels at 3 months of clinical remission predicted progression at 18 months.

Uterine cancer: PGRN protein staining was observed in the cytoplasm of tumor cells and stromal cells in endometrial cancer samples [36]. In endometrial cancer, PGRN protein expression was not associated with poor overall survival or known biomarkers of prognosis, including stage and grade. Approximately two thirds of the cancers co-expressed PGRN and ER. Elevated PGRN levels were observed in uterine leiomyosarcomas by immunohistochemical analysis, and the PGRN protein expression positively correlated with histological grades of tumors [37]. Those findings suggested that PGRN might be used to become a specific diagnostic marker for uterus leiomyosarcomas.

Prostate cancer: High levels of PGRN immunostaining were observed in prostate cancer tumor cells both among prostatic intraepithelial neoplasia (PIN) specimens and invasive cancer specimens $[38,39]$. PGRN was absent or showed low level staining in prostatic glands of normal tissues. However, PGRN expression level has no significant correlation with pathological stage, Gleason score, the status of lymph node metastasis, extraprostatic extension, perineural invasion, surgical margins, and vascular invasion. The elevated levels of PGRN staining in PIN suggest that elevated PGRN production may occur in the earlier stages of prostate cancer development.

Bladder cancer : Monami et al. [40] found that PGRN expression was detectable in both bladder cancer cells and normal bladder urothelium, and suggested that PGRN may play important roles in 
carcinogenesis as well as regulation of normal physiological activity. Significantly higher immunostaining of PGRN was observed in invasive bladder tumors as compared with normal bladder tissues [41]. Lovat et al. [41] analyzed PGRN mRNA expression in bladder cancer using microarray database and found that overexpression of PGRN was observed in primary bladder cancers. PGRN mRNA expression levels was higher in high-grade bladder cancer than that of low-grade bladder cancer, and overexpression of PGRN were observed in patients who died after 5 years of follow-up compared with those alive after 5 years of follow-up treatment [41,42]. PGRN levels in voided urine samples from bladder cancer patients revealed that PGRN level was significantly higher in patients with malignant lesions compared to healthy individuals [43].

Kidney cancer: Elevated PGRN levels were detected in renal cell carcinoma samples using Western and immunohistochemical analysis [44]. PGRN expression correlated positively with the histological grades of tumors.

Liver cancer: Overexpression of PGRN was observed in a majority of hepatocellular carcinoma (HCC) patients using immunohistochemical methods on liver tumor specimens $[45,46]$. Patients with high PGRN content in clinical specimens showed characteristics of aggressive HCC, such as large size of tumor and venous infiltration [46]. PGRN protein is commonly observed in the cytoplasm of HCC tumor cells $[46,47]$. Cheung et al. $[46,48]$ also reported that strong expression level of PGRN is significantly associated with early recurrence after curative resection and suggested that overexpression of PGRN is a predictive biomarker of poor outcome for HCC.

Esophageal cancer: PGRN protein is highly expressed in both cytoplasm and nuclei of esophageal squamous cell carcinoma cells [49]. Positive staining was also observed in stroma, including sporadic interstitial cells, esophageal glands, and vascular endothelial cells. In well differentiated tumors, positive staining of PGRN is uneven, whereas uniformly strongly positive staining was found in poorly differentiated tumors. PGRN positive staining was almost un-detectable in normal esophageal endothelium. PGRN positive staining was correlated with tumor infiltration depth and TNM classification.

Gastric cancer: Serological identification of tumour antigens by recombinant expression cloning identified $G R N$ gene as overexpressed in gastric cancer [50]. PGRN was highly expressed in gastric cancer specimens with 2.3-2.9 folds of difference compared with the adjacent non-cancerous tissues. Using immunohistochemical analysis, PGRN protein expression was almost absent in normal gastric tissues, however $88 \%$ of cells were PGRN-positive in gastric cancer biopsy specimens [51]. This may not be specific for neoplasia since increased mucosal PGRN staining was also observed in gastritis [51].

Laryngeal cancer: Using immunohistochemical staining on samples from primary laryngeal cancer and adult laryngeal papilloma as well as laryngeal leukoplakia, PGRN protein expression was found to be significantly higher in the cytoplasm of laryngeal squamous cell carcinomas (LSCC) than those of the other lesions [52]. Overexpression of PGRN mRNA levels was also observed in LSCC tissues.

Lung cancer: PGRN was investigated as a new candidate lung cancer biomarker by one large cohort study but was not found informative as a lung cancer biomarker [53].

Brain cancer: PGRN is consistently overexpressed in human glioblastomas tumors compared with normal brain samples [54]. Using microarrays, Martert et al. [55] investigated gene expression profiles between primary human glioblastoma multiforme (GBM) tumors and normal brain tissues, and found that PGRN is upregulated in GBM tumors. PGRN expression was detected in $36.7 \%$ of meningioma tumor samples using reverse transcription-polymerase chain reaction (RT-PCR) [56]. The average size $\left(51.5 \pm 5.9 \mathrm{~cm}^{3}\right)$ of tumors with PGRN expression was significantly larger than the mean meningioma volume $\left(24.9 \pm 2.8 \mathrm{~cm}^{3}\right)$ of PGRN-negative tumors. The mean area of peritumoral brain edema which is associated with malignant development, was significantly larger in PGRN positive tumors than that in tumors with absence of PGRN.

Myeloma and leukemia: PGRN mRNA and protein expression were observed in multiple myeloma (MM) cell lines, and the immunohistochemical analysis showed PGRN positive staining in bone marrow smears from MM patients but no detectable PGRN staining in bone marrow biopsy samples from patients in remission [57]. Larramendy and et al. [58] found that PGRN expression was significantly down-regulated in acute myeloid leukemia as assessed by cDNA microarray analysis.

Infectious agents, progranulin and cancer: In south-east Asia, chronic infection with the liver fluke Opisthorchis viverrini (O. viverrini) is a major risk factor for bile ducts cancer or cholangiocarcinoma. Screening genes from the transcriptome [59] or the excretory/secretory proteome [60] of O. viverrini that are associated with cancer in humans identified a parasite homologue of human PGRN. PGRN was secreted by the liver flukes and released into bile ducts to stimulate proliferation of biliary epithelial cells to promote host cells to turnover with the potential to increase susceptibility to ductal carcinogenesis $[59,60]$. Patients infected by Helicobacter pylori (H. pylori) have a higher than average risk of developing gastric cancer [61]. H. pylori infection was found to induce overexpression of PGRN at both mRNA and protein levels in gastric epithelial cells [51] which may contribute to development of gastric cancers.

\section{PGRN promotes tumorigensis in experimental models}

Given that PGRN is often over-expressed in cancers, and that PGRN levels are associated with poor outcome, does PGRN have a functional role in carcinogenesis? Over production of PGRN frequently confers a more aggressive phenotype on cancer cells in vitro, as assessed by parameters such as anchorage-independent growth, improved survival or invasion assays (for examples, see refs [34,36,38,40,45,46,57,62-66]). Increasing the production of PGRN in poorly tumorigenic cells often results in a more malignant phenotype following transplantation into mice. The over-production of PGRN in the estrogen-dependent breast cancer cell line MCF7, for example, results in increased tumorigenicity, estrogen-independence and resistance to the estrogen receptor blocking drug Tamoxifen [63,67]. Similarly, PGRN over-production confers greater tumorigenicity upon liver [46], ovarian [68] and endometrial cancer cells [36] when grown as xenografts in mice. SW13 cells, which derive from an adrenal carcinoma, are highly sensitive to the proliferative effects of PGRN [3,65]. The parental SW13 cells are poorly tumorogenic in vivo, however by over-producing PGRN they become highly tumorigenic in mice [3]. SW13 cells have defects in the p53 [69] and $\mathrm{Rb}$ [70] tumor-suppressor systems, and exhibit little detectable expression of cyclin-dependent kinase inhibitors such as p21 or p16 ${ }^{\text {ink4a }}$ [69]. Presumably the loss of function of tumor suppressors such as p53 in SW13 cells removes the brakes on the cell cycle, while PGRN provides the acceleration that drives them towards a more malignant state. 
Primary cultures of human cells are more difficult to transform by direct gene transfer than rodent cells [71]. Specifically, there is a requirement for genes that prevent senescence (immortalizing genes) to cooperate with genes that activate the RAS-mediated cell signaling pathway and thereby stimulate proliferation [72-74]. In particular, RAS-mediated activation of guanine nucleotide exchange factors for the Ral small GTPases appears critical in the transformation of human cells [75]. Very few genes can be successfully substituted for mutant RAS in these primary cell transformation assays. Examples inhuman ovarian surface epithelial (OSE) cells include the oncogenic epidermal growth factor receptor-family member HER-2 [76]. Importantly, PGRN is also able to substitute for oncogenic RAS in the transformation of immortalized human OSE cells [33] as well as in human uterine smooth muscles cells [37]. These primary cells [33,37], that were first immortalized by expression of telomerase (hTERT) and SV40 large-T antigen (SV40) and then forced to express the GRN gene at high levels, were strongly tumorigenic when transplanted into athymic mice. Immortalized cells expressing hTERT and SV40, but that did not over-express the GRN gene, were not tumorigenic [37,33]. Similarly the expression of the GRN gene on its own did not transform the primary cells. The expression of SV40 impedes inhibitory control mechanisms on the cell cycle exerted by the p53 and Rb systems, as well as inhibiting the protein phosphatase $2 \mathrm{~A}$ [77] and prevents mitogenmediated senescence, while increased expression of the GRN gene appears to provide the thrust that drives the immortalized, but noncancerous cells towards a tumorigenic phenotype. The ability of PGRN to replace oncogenic RAS in transforming immortalized cells suggests that it is a highly oncogenic protein. This interpretation, that PGRN can transform immortalized (pre-cancerous) cells is supported in other systems including the non-transformed but immortal renal epithelial cell line, MDCK, which becomes highly anchorage-independent when engineered to produce elevated levels of PGRN [3].

Just as the over-expression of PGRN promotes a more proliferative and tumorigenic phenotype, the reduction of PGRN mRNA levels may reduce proliferation in vitro and tumor growth in vivo. In tissue culture, proliferation and anchorage-independence can be inhibited by targeting PGRN mRNA in breast cancer [78], SW13 adrenal carcinomal cells [3], MDCK kidney epithelial cells [3], laryngeal cancer cells [52], prostate cancer cells [38], ovarian cancer cells [32,79] and liver cancer cells [80]. PC cells, which are a highly tumorigenic murine teratoma-derived cell line, secrete a growth factor, called PC cell-derived growth factor that is identical in structure to murine PGRN [6]. Targeting PGRN mRNA levels in PC cells abolished their in vivo tumorigenicity [81]. Lowering the mRNA levels of PGRN in other tumorigenic cell lines, including the breast cancer line MDA-MB-468 [78], the laryngeal carcinomal cell line Hep-2 [52], and liver cancer cells [80] also results in profound inhibition of tumor growth in mice. Monoclonal antibodies against PGRN are effective at inhibiting tumor growth of transplanted liver cancer cells in nude mice, and work by targeting both the growth of the tumor cells directly, but also by suppressing tumor angiogenesis [45]. Taken together, these results suggest that progranulin is both sufficient to stimulate a more malignant phenotype in poorly tumorigenic or immortalized cells; that it is necessary for tumor growth in some aggressive cancers, and that it has promise as a molecular target in the development of novel anti-cancer therapies.

\section{PGRN and the tumor stroma}

It is well established that the non-transformed mesenchymal cells that surround the cancer and form the tumor stroma have a crucial role in the growth and metastasis of many cancer types [82]. In most healthy tissues, mesenchymal cells such as fibroblasts or endothelial cells express the GRN gene at low or negligible levels [83]. However, in the fibroblasts of the tumor stroma and tumor capillary endothelial cells, GRN gene expression may be abundant. This is well documented for ovarian cancers, where approximately half of the tumors examined displayed PGRN-staining in stromal fibroblasts and in two thirds of the tumors capillaries were PGRN positive [31]. This has important consequences since, as noted above the presence of ovarian stromal PGRN correlated with a poorer outcome [31]. In breast cancer cells PGRN stimulated increased production of the angiogenic proteins vascular endothelial growth factor (VEGF) [84,67] and angiopoietin [67]. Esophageal squamous cell carcinomas exhibited a positive correlation between PGRN and VEGF levels, with the levels of both proteins correlating with microvessel density [49]. Blockade of PGRN by monoclonal antibodies in HCC xenografts resulted in a decrease in tumor microvessel density, which was attributed to reduced production of VEGF [45]. PGRN may also have a direct effect on angiogenesis that is independent of VEGF production since it stimulated the proliferation and migration of endothelial cells in culture [20].

McAllister and colleagues have recently proposed a novel mechanism for tumor stroma formation where an initial robust breast cancer mass, the instigator, stimulates the formation of reactive tumor stroma in a second poorly-growing or indolent tumor located at a distant anatomical site $[85,25]$. The instigating tumors secrete the prometastatic protein osteopontin that activates the migration of a population of Sca $1^{+} \mathrm{cKit}$ hematopoietic stem cells from the bone marrow to the quiescent tumor [85]. Upon taking up residence in the indolent tumor the $\mathrm{Sca} 1^{+} \mathrm{cKit}$ bone marrow cells secrete PGRN, which in turn activates the growth of a fibroblastic stroma around the quiescent cancer cells [25]. The cancer cells then proliferate under the influence of the recently formed stroma to create new tumor masses [25]. Many of the details of how PGRN contributes to the formation and activity of the stroma remain unclear, but in principle the PGRNsecreting $\mathrm{Scal}^{+}{ }^{+} \mathrm{Kit}-$ bone marrow cells might initiate the formation of the tumor stroma, while at a later stage intrinsic PGRN production by stromal fibroblasts may supplement or take over the role of the bone marrow cells in promoting and maintaining the growth of the tumor stroma.

\section{PGRN, cell survival and drug resistance}

PGRN is a putative survival factor for normal and cancer cells in vitro. This may contribute to the overall growth of the PGRN-sensitive tumors, and may complicate cancer therapy since PGRN appears to confer increased resistance to several classes of anti-cancer drugs. PGRN prevents anoikis in cancer cells [65], a form of apoptosis that occurs when cells detach from their basement membrane [86]. Immunoneutralization of PGRN in ovarian cancer cells results in enhanced apoptosis as assessed by increased caspase- 3 activation and poly(ADP-ribose) polymerase cleavage [79]. PGRN inhibits metabolicstress apoptosis in non-transformed fibroblasts [87] and cultured neurons $[88,89]$. Recent work in C. elegans and with macrophage from Grn knockout mice revealed that in the absence of PGRN the rate of phagocytosis of apoptotic cells increased [90], suggesting that PGRN may inhibit apoptotic clearance of injured or diseased cells.

Endocrine therapy is a mainstay in the treatment of estrogen receptor (ER)-positive breast cancer, with selective estrogen receptor modulators such as tamoxifen [91] playing an essential role in the treatment of ER-positive breast cancer. Over-production of PGRN in estrogen receptor-positive MCF-7 cells induced tamoxifen-resistance 
$[63,67]$. The activation of mitogen-activated protein kinase (MAPK) signaling pathways in breast cancer cells by the over-production of growth factors or growth factor receptors promotes proliferation without requiring ER-mediated growth signaling [92]. This confers resistance to antiestrogens [93] thereby rendering antiestrogen therapy in effective [92]. In this regard, PGRN markedly increased MAPK activity in breast cancer cell lines [63]. Moreover, PGRN treatment in MCF-7 cells inhibited the tamoxifen-induced down-regulation of bcl-2 [94]. PGRN may in addition interfere with aromatase therapy since PGRN inhibited the efficacy of the aromatase inhibitor letrozole in a human breast cancer cell line [95]. PGRN expression level was significantly increased in letrozole resistant tumor cells compared with letrozole sensitive cancer cells [95]. Other hormone-based therapies may also be sensitive to PGRN production. For example, PGRN decreased apoptosis induced by the synthetic glucocorticoid dexamethasone (which is used in the therapeutic regimen of multiple myeoloma) in a dexamethasone-sensitive multiple myeloma cell line [96].

Ovarian cancer cells that constitutively over-produce PGRN were resistant to the platinum containing drug cisplatin [68] (which is used to treat ovarian cancer [97]). However, ovarian cancer stromal cells that were sampled after chemotherapy with platinum drugs tended to show reduced staining for PGRN compared to comparable tissues inspected before treatment [31] suggesting a level of complexity in the drug-PGRN relationship. The recurrence of liver cancer and chemoresistance are huge obstacles to provide curative treatment for patients $[48,98,99]$. In hepatic cancer stem cells [48], inhibiting the expression of GRN gene sensitized the HCC cells to chemotherapy [48], whereas elevated production of PGRN led to resistance to cisplatin and doxorubicin. In chemoresistant HCC cells, PGRN levels were positively associated with expression of the adenosine triphosphatedependent binding cassette $\mathrm{ABCB} 5$ which is a drug transporter in liver cancer cells [48]. The interaction between PGRN and ABCB5 may provide a potential mechanism by which PGRN confers drug resistance. Chemotherapeutic resistance in non-small cell lung cancer (NSCLC) increased with the expression of PGRN [100]. Hu and et al. $[101,102]$ studied the correlation between $G R N$ expression in serum or tumor samples and chemotherapeutic sensitivity in NSCLC and found significant higher expression of PGRN in chemoresistant patients than in chemosensitive patients. They concluded that PGRN expression was significantly associated with response of chemotherapy and PGRN might become a biomarker to evaluate chemotherapeutic sensitivity and predict medical prognosis among NSCLC patients.

\section{The mechanism of action of PGRN}

A functional receptor for PGRN has not been identified, although chemical cross-linking experiments show that PGRN [103], its constituent grn/epi peptides [104] and TGFe bind in a specific fashion to cell surface proteins [105]. Scatchard analyses indicated that both for PGRN [103] or grn/epi peptides [104] at least two classes of PGRN binding site exist, one of low affinity but high abundance together with other sites of low abundance but high affinity. PGRN binds with sortilin on cell surfaces which is likely to be part of a protein turnover mechanism [106]. PGRN also binds to and inhibits the TNF-receptors $[106,107]$ and associates with the Toll-like receptor-9 [108]. These interactions are important in the regulation of inflammation. Other binding partners for PGRN include non-receptor extracellular matrix proteins such as perlecan [109] and cartilage oligomeric matrix protein [110]. The binding of PGRN to these extracellular matrix proteins modifies the biological action of PGRN either blunting or enhancing the combined proliferative effects of the PGRN-matrix protein pair [110].

PGRN stimulates the MAPK and phosphatidylinositol 3-kinase (PI3K) pathways in cancer cells, as well as in non-transformed fibroblasts and neurons [20,34,38,40,45,57,62,63,65,66,111,112]. Both the MAPK and PI3K signaling systems are essential for PGRN mediated cell division, survival and invasion [65]. Interestingly different cells may show different signaling responses to PGRN, for example, bladder cancer cells show clear activation of MAPK in response to PGRN but little or no PI3K response [38]. Significantly, the bladder cells respond well to the motility and invasive activity of PGRN but not to its proliferative actions [38]. PGRN signaling may interact with integrin signaling pathways through focal adhesion kinase (FAK) $[20,65]$. In bladder cells PGRN-stimulated the formation of intracellular complexes of MAPK, paxillin and FAK [40] thereby linking the ERK and FAK signaling machinery. FAK is essential for growth factor and integrin-regulated cellular motility (reviewed in [113]). In addition to promoting motility-related signal transduction events, PGRN stimulates the expression of matrix metalloproteinases (MMPs) in cancer cells, including MMP2, MMP9, MMP13 and MMP17 $[65,84,114]$ which probably contributes to the migratory properties of cells stimulated by PGRN. Downstream effects of PGRN signaling include expression of proteins of the cell cycle such as cyclin D1 $[62,63,114]$, and cyclin B [62], the phosphorylation of other signaling intermediates such as glycogen synthase kinase beta [111,112], and the activation of transcriptional regulators such as nuclear factor kappa-B in myeloma cells [96] and JunB in chondrocytes [16].

The activation of the MAPK and PI3K signaling systems are characteristic of all the classic growth factors, however differences between the signaling properties of PGRN and those of conventional growth factors have been postulated. Non-transformed fibroblasts require stimulation by two independent growth factors in order to complete the cell cycle under serum free conditions [115]. One of these signals is provided by an insulin-like growth factor (IGF), while the other signal may be provided by one of a number of growth factors including members of the platelet-derived growth factor (PDGF), fibroblast growth factor (FGF) or epidermal growth factor (EGF) families. Genetically deleting the receptor for IGF-1 prevents the mitotic activity of the IGFs on murine fibroblasts, but also inhibits all other growth conventional factors from stimulating proliferation [116]. PGRN, however, retains the ability to promote cell division in IGF-I receptor negative fibroblasts under serum free conditions, indeed it is the only extracellular protein known to do so $[62,117]$. It is thought that rather than any ability of PGRN to stimulate distinct signaling pathways it has this property because it stimulates a more prolonged activation of proteins in the MAPK and PI3K pathways than do classic growth factors such as PDGF or EGF [62]. Unlike their wild-type counterparts, IGF-I receptor negative fibroblasts cells ( $\mathrm{R}^{-}$cells) are not transformed by most oncogenes [116], although they are transformed by constitutively active mutant G-protein G-alpha 13 [118], and become sensitive to the transformative activity of SV40 T as they age, possibly due to enhanced expression of the EGF-receptor HER-3 [119]. Whether PGRN signaling also interacts with G-alpha 13 or HER-3 is unknown. The ability for PGRN to promote proliferation in cells such as the R' cells, that are otherwise refractory to growth factor stimulation or the transformative effects of oncogenes, is worrisome in that it provides a putative pathway through which cells could escape the therapeutic effects of anti-cancer drugs that work by targeting growth 
Citation: Zhang Y, Bateman A (2011) The Glycoprotein Growth Factor Progranulin Promotes Carcinogenesis and has Potential Value in Anti-cancer Therapy. J Carcinogene Mutagene S2:001. doi:10.4172/2157-2518.S2-001

factors and their receptors. A summary of PGRN's role and mechanism in cancer is given in Figure 2.

Although the focus in this article is on PGRN as a mitogen, a cell survival factor and a promoter of invasion, it may have many other biological effects, some of which could contribute to tumor growth. PGRN is anti-inflammatory [17-19], which might in principle effect the host's immune response to developing tumors. Further, PGRN may regulate protein turnover since the over-production of PGRN by HeLa cells, or treatment of the cells with PGRN-conditioned medium, stimulates the formation of more and larger lysosomes [120]. Consistent with these observations, treatments that promote lysosome formation, such as sucrose or the expression of the transcription factor EB, stimulate GRN gene expression [120]. PGRN is reported to interact with intracellular proteins such as cyclin-T [121-123] which may modulate transcription. It remains uncertain if these interactions contribute to carcinogenesis, but they clearly provide additional possibilities through which the expression of the GRN gene could modulate cell function.

\section{Regulation of GRN gene expression}

At present there is no evidence that the GRN gene is amplified during tumorigenesis, suggesting instead that the elevated expression of the $G R N$ gene in cancer cells results from changes in its regulation rather than an increased gene copy number. Mechanisms for the regulation of the GRN gene include mRNA stability [124], microRNAs [125128], the action of RNA binding proteins [129], signal transduction by MAPK signaling [79], nuclear hormone receptors [130], and by physiological parameters such as hypoxia $[87,131]$.

GRN gene expression is stimulated by nuclear receptor hormones such as retinoic acid [124] and, in breast cancer cells [130], endometrial cancer cells [36] and the hypothalamus [132] by estrogen. Importantly, the increase in GRN expression that follows exposure to estrogen in breast cancer cells may set in motion a PGRN autocrine growth stimulatory loop [133] since the mitogenic activity of estrogen on MCF7 breast cancer cells in culture is inhibited by the immunoneutralization of PGRN [63], while enhanced GRN expression in breast cancer cells
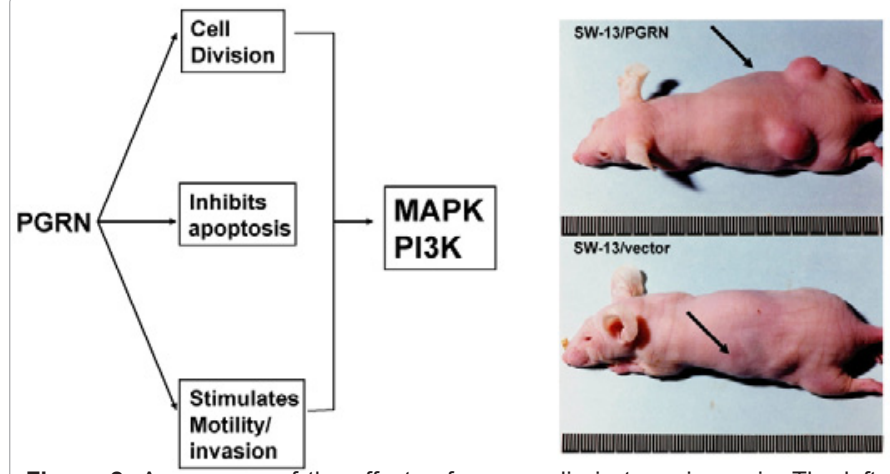

Figure 2: A summary of the effects of progranulin in tumorigenesis. The left panel shows that PGRN stimulates cancer cell migration, survival and mitosis through MAPK and PI3K dependent mechanisms. Although not shown here, progranulin may stimulate the proliferation and activation of tumor resident fibroblasts in the stroma possibly through activating similar biological responses. The right panel shows the growth of SW-13 tumors in athymic nude mice eight weeks after subcutaneous injection into both flanks. Cells that were engineered to over produce progranulin (SW-13/PGRN) formed large tumors (arrow), while control cells (SW-13/vector) gave either small tumor growths (arrow) or undetectable tumor growth. (The scale marker is in $\mathrm{cm}$ with $2 \mathrm{~mm}$ gradations) For details see ref [3] enables them to form tumors independently of estrogen [67].

Mitogens other than estrogen may also stimulate GRN gene expression. The proliferative actions of endothelin and lysophospahtidic acid (LPA) on ovarian cells were blocked when PGRN was immunoneutralized, suggesting that PGRN acts as an autocrine signal for these mitogens [79]. Both endothelin and LPA promote the expression of the GRN gene [79] through the activation of MAPK by a protein kinase A, calcium and cyclicAMP-dependent mechanism [79]. The up-regulation of GRN gene expression by MAPK may be widespread since it is also reported in neuroblastoma cell lines [131] and in gastric mucosa [51] in response respectively to hypoxia or the presence of the gastritis causing bacterium $H$. pylori.

Differentiation agents such as retinoic acid and dimethylsulfoxide increase GRN mRNA expression in myelogenous leukemias [124]. This revealed for the first time the importance and complexity of PGRN mRNA stability in GRN gene regulation. In a progranulocytic cell line, for example, differentiation agents promoted faster GRN mRNA turnover, resulting in a rapid but transient increase in PGRN mRNA following stimulation. In contrast, in a promonocytic cell line identical treatments slowed the rate of PGRN mRNA turnover [124] leading to a slower and more prolonged elevation of the GRN mRNA level. Genetic studies on GRN in neurodegenerative disease proved that microRNAs (miRs), in particular miR-659 [125] and miR-107 [127] are critical negative regulators of PGRN mRNA levels. This extends to cancer cells, where miRs belonging to the miR-15/107 gene group were found to suppress PGRN mRNA levels in prostate cancer cells [128]. This may be functionally significant since in leukemic and prostate cancers low miR-15/107 correlates with high GRN expression [128]. GRN gene expression may be negatively regulated by the p53 tumor suppressor system. Restoring a functional p53 to the malignant glioma cell line LNZ308 which has lost both p53 allelles, results in an decreased secretion of PGRN [134]. Against this however, in HCCs higher GRN expression correlated with higher levels of wild-type but not mutated p53 [47] suggesting a complex relationship between GRN gene expression and P53. Additional control of PGRN mRNA expression through RNAbinding proteins has recently been established. The dying neurons of patients with $G R N$ gene mutations accumulate intracellular aggregates of a cleaved and ubiquitinated DNA-and-RNA binding protein called the TAR DNA Binding protein or TDP-43 [21,22]. The functional relationship between PGRN and TDP-43 in neurodegenerative disease is not well understood, but in normal brain tissue TDP-43 binds to and decreases PGRN mRNA levels [129]. Whether disruptions in the binding of TDP-43 (or similar RNA-binding proteins) to PGRN mRNA have a role in carcinogenesis is unknown.

The proteolysis of secreted PGRN provides an additional control over PGRN levels. PGRN is digested by matrix metalloproteinases (MMPs) including MMP-9 and MMP-14 [135,136] as well as ADAMTS-7 (a member of the "A Disintegrin And Metalloproteinase with Thrombospondin Motifs" gene family) [137]. During inflammation neutrophil-derived enzymes such as elastase and proteinase-3 digest PGRN down to its constituent $6 \mathrm{kDa}$ grn/epi peptides, some of which have biological activity in, for example, the regulation of inflammation $[17,138]$. This proteolysis is prevented by the secretory leukocyte protease inhibitor (SLPI) which, in addition to inhibiting elastase enzyme activity, physically binds to PGRN and protects it against enzymatic cleavage [17]. Intriguingly there is strong evidence that SLPI and PGRN act in concert to promote ovarian tumor cell mitosis and survival $[139,140]$. Other proteins in addition to SLPI 
Citation: Zhang Y, Bateman A (2011) The Glycoprotein Growth Factor Progranulin Promotes Carcinogenesis and has Potential Value in Anti-cancer Therapy. J Carcinogene Mutagene S2:001. doi:10.4172/2157-2518.S2-001

may protect PGRN from enzymatic degradation, including extracellular matrix proteins such as cartilage oligomeric matrix protein [141], and serum proteins such as high density lipoprotein [142]. The turnover of PGRN at the cell surface is controlled by Sortilin [106,107]. Sortilin was originally identified as a regulator of lysosomal enzyme trafficking [143] and is critical in controlling extracellular PGRN levels in neuronal cells $[106,107,144]$. Whether it has comparable functions in cancer cells is at present unknown. Taken together, the levels and activity of PGRN in vivo are likely to depend first on the intra- and extracellular factors that regulate PGRN mRNA levels, followed by complex extracellular interactions between PGRN, proteolytic enzymes, and stabilizing proteins such as SLPI that inhibit the proteolysis of PGRN.

\section{Conclusion}

PGRN levels are often highly elevated in tumors at many anatomical sites compared to the equivalent normal tissue. PGRN may therefore have potential as a biomarker for disease outcome. PGRN acts as a mitogen, a cell survival factor and a promoter of invasion for a variety of cancer cells. PGRN may have other biological effects that contribute to tumor growth, including the formation of the tumor stroma, the induction of drug resistance, and anti-inflammatory actions. Given the frequency with which PGRN expression occurs in cancers and its tumorigenic biological actions, there is a strong likelihood that studies of the cellular and molecular mechanisms of PGRN action in tumor formation will supply innovative strategies for the development of novel anti-cancer therapies.

\section{Acknowledgements} Institute.

Funding to $A B$ was provided by the Canadian Cancer Society Research

\section{References}

1. Halper J (2010) Growth factors as active participants in carcinogenesis: a perspective. Vet Pathol 47: 77-97.

2. Siegel R, Ward E, Brawley O, Jemal A (2011) Cancer statistics, 2011: The impact of eliminating socioecnomic and racial disparities on premature cancer deaths. CA Cancer J Clin 61: 212-236.

3. He Z, Bateman A (1999) Progranulin gene expression regulates epithelial cell growth and promotes tumor growth in vivo. Cancer Res 59: 3222-3229.

4. Bhandari V, Daniel R, Lim PS, Bateman A (1996) Structural and functional analysis of a promoter of the human granulin/epithelin gene. Biochem $\mathrm{J} 319$ : 441-447.

5. Plowman GD, Green JM, Neubauer MG, Buckley SD, McDonald VL, et al. (1992) The epithelin precursor encodes two proteins with opposing activities on epithelial cell growth. J Biol Chem 267: 13073-13078.

6. Zhou J, Gao G, Grabb JW, Serrero G (1993) Purification of an autocrine growth factor homologous with mouse epithelin precursor from a highly tumorigenic cell line. J Biol Chem 268: 10863-10869.

7. Baba T, Hoff HB 3rd, Nemoto H, Lee H, Orth J, et al. (1993) Acrogranin, an acrosomal cysteine-rich glycoprotein, is the precursor of the growth-modulating peptides, granulins, and epithelins, and is expressed in somatic as well as male germ cells. Mol Reprod Dev 34: 233-243.

8. Parnell PG, Wunderlich J, Carter B, Halper J (1992) Transforming growth factor e: amino acid analysis and partial amino acid sequence. Growth Factors 7: 6572.

9. Songsrirote K, Li Z, Ashford D, Bateman A, Thomas-Oates J (2010) Development and application of mass spectrometric methods for the analysis of progranulin N-glycosylation. J Proteomics 73: 1479-1490.

10. Bateman A, Belcourt D, Bennett H, Lazure C, Solomon S (1990) Granulins, a novel class of peptide from leukocytes. Biochem Biophys Res Commun 173: 1161-1168.

11. Shoyab M, McDonald VL, Byles C, Todaro GJ, Plowman GD (1990) Epithelins 1 and 2: isolation and characterization of two cysteine-rich growth-modulating proteins. Proc Natl Acad Sci USA 87: 7912-7916.

12. Bhandari V, Palfree RG, Bateman A (1992) Isolation and sequence of the granulin precursor cDNA from human bone marrow reveals tandem cysteinerich granulin domains. Proc Natl Acad Sci USA 89: 1715-1719.

13. Sparro G, Galdenzi G, Eleuteri AM, Angeletti M, Schroeder W, et al. (1997) Isolation and $\mathrm{N}$-terminal sequence of multiple forms of granulins in human urine. Protein Expr Purif 10: 169-174.

14. Diaz-Cueto L, Stein P, Jacobs A, Schultz RM, Gerton GL (2000) Modulation of mouse preimplantation embryo development by acrogranin (epithelin/granulin precursor). Dev Biol 217: 406-418.

15. Qin J, Diaz-Cueto L, Schwarze JE, Takahashi Y, Imai M, et al. (2005) Effects of progranulin on blastocyst hatching and subsequent adhesion and outgrowth in the mouse. Biol Reprod 73: 434-442.

16. Feng JQ, Guo FJ, Jiang BC, Zhang Y, Frenkel S, et al. (2010) Granulin epithelin precursor: a bone morphogenic protein 2-inducible growth factor that activates Erk1/2 signaling and JunB transcription factor in chondrogenesis. FASEB J 24 1879-1892.

17. Zhu J, Nathan C, Jin W, Sim D, Ashcroft GS, et al. (2002) Conversion of proepithelin to epithelins: roles of SLPI and elastase in host defense and wound repair. Cell 111: 867-878.

18. Yin F, Banerjee R, Thomas B, Zhou P, Qian L, et al. (2010) Exaggerated inflammation, impaired host defense, and neuropathology in progranulindeficient mice. J Exp Med 207: 117-128.

19. Tang W, Lu Y, Tian QY, Zhang Y, Guo FJ, et al. (2011) The growth factor progranulin binds to TNF receptors and is therapeutic against inflammatory arthritis in mice. Science 332: 478-484.

20. He Z, Ong CH, Halper J, Bateman A (2003) Progranulin is a mediator of the wound response. Nat Med 9: 225-229.

21. Cruts M, Gijselinck I, van der Zee J, Engelborghs S, Wils H, et al. (2006) Nul mutations in progranulin cause ubiquitin-positive frontotemporal dementia linked to chromosome 17q21. Nature 442: 920-924.

22. Baker M, Mackenzie IR, Pickering-Brown SM, Gass J, Rademakers R, et al. (2006) Mutations in progranulin cause tau-negative frontotemporal dementia linked to chromosome 17. Nature 442: 916-919.

23. Serrero G, loffe OB (2003) Expression of PC-cell-derived growth factor in benign and malignant human breast epithelium. Hum Pathol 34: 1148-1154.

24. Song H, Shi L, Liu C, Huang T (2009) Expression of PC-cell-derived growth factor in breast cancer. Front Med China 3: 426-430.

25. Elkabets M, Gifford AM, Scheel C, Nilsson B, Reinhardt F, et al. (2011) Human tumors instigate granulin-expressing hematopoietic cells that promote malignancy by activating stromal fibroblasts in mice. J Clin Invest 121: 784-799.

26. Sorlie T (2004) Molecular portraits of breast cancer: tumour subtypes as distinct disease entities. Int J Cancer 40: 2667-2675

27. Hudis CA,Gianni L (2011) Triple-negative breast cancer: an unmet medical need. Oncologist 16 Suppl 1: 1-11.

28. Tkaczuk KR, Yue B, Zhan M, Tait N, Yarlagadda L, et al. (2011) Inceased Circulating Level of the Survival Factor GP88(Progranulin) in the Serum of Breast Cancer Patients When Compared to Healthy Subjects. Breast Cancer 5: 155-162.

29. Asaka S, Fujimoto T, Akaishi J, Ogawa K, Onda M (2006) Genetic prognostic index influences patient outcome for node-positive breast cancer. Surg Today 36: 793-801.

30. Jemal A, Bray F, Center MM, Ferlay J, Ward E, et al. (2011) Global cancer statistics. CA Cancer J Clin 61: 69-90

31. Davidson B, Alejandro E, Florenes VA, Goderstad JM, Risberg B, et al. (2004) Granulin-epithelin precursor is a novel prognostic marker in epithelial ovarian carcinoma. Cancer 100: 2139-2147.

32. Jones MB, Michener CM, Blanchette JO, Kuznetsov VA, Raffeld M, et al. (2003) The granulin-epithelin precursor/PC-cell-derived growth factor is a growth factor for epithelial ovarian cancer. Clin Cancer Res 9: 44-51.

33. Miyanishi M, Mandai M, Matsumura N, Yamaguchi K, Hamanishi J, et al. 
Citation: Zhang Y, Bateman A (2011) The Glycoprotein Growth Factor Progranulin Promotes Carcinogenesis and has Potential Value in Anti-cancer Therapy. J Carcinogene Mutagene S2:001. doi:10.4172/2157-2518.S2-001

(2007) Immortalized ovarian surface epithelial cells acquire tumorigenicity by Acrogranin gene overexpression. Oncol Rep 17: 329-333.

34. Cuevas-Antonio R, Cancino C, Arechavaleta-Velasco F, Andrade A, Barron L, et al. (2010) Expression of progranulin (Acrogranin/PCDGF/Granulin-Epithelin Precursor) in benign and malignant ovarian tumors and activation of MAPK signaling in ovarian cancer cell line. Cancer Invest 28: 452-458.

35. Han JJ, Yu M, Houston N, Steinberg SM, Kohn EC (2011) Progranulin is a potential prognostic biomarker in advanced epithelial ovarian cancers. Gynecol Oncol 120: 5-10.

36. Jones MB, Houwink AP, Freeman BK, Greenwood TM, Lafky JM, et al (2006) The granulin-epithelin precursor is a steroid-regulated growth factor in endometrial cancer. J Soc Gynecol Investig 13: 304-311.

37. Matsumura N, Mandai M, Miyanishi M, Fukuhara K, Baba T, et al. (2006) Oncogenic property of acrogranin in human uterine leiomyosarcoma: direct evidence of genetic contribution in in vivo tumorigenesis. Clin Cancer Res 12: $1402-1411$.

38. Monami G, Emiliozzi V, Bitto A, Lovat F, Xu SQ, et al. (2009) Proepithelin regulates prostate cancer cell biology by promoting cell growth, migration, and anchorage-independent growth. Am J Pathol 174: 1037-1047.

39. Pan CX, Kinch MS, Kiener PA, Langermann S, Serrero G, et al. (2004) PC cell-derived growth factor expression in prostatic intraepithelial neoplasia and prostatic adenocarcinoma. Clin Cancer Res 10: 1333-1337.

40. Monami G, Gonzalez EM, Hellman M, Gomella LG, Baffa R, et al. (2006) Proepithelin promotes migration and invasion of 5637 bladder cancer cells through the activation of ERK $1 / 2$ and the formation of a paxillin/FAK/ERK complex. Cancer Res 66: 7103-7110.

41. Lovat F, Bitto A, Xu SQ, Fassan M, Goldoni S, et al. (2009) Proepithelin is an autocrine growth factor for bladder cancer. Carcinogenesis 30: 861-868.

42. Blaveri E, Simko JP, Korkola JE, Brewer JL, Baehner F, et al. (2005) Bladder cancer outcome and subtype classification by gene expression. Clin Cancer Res 11: 4044-4055.

43. Selmy MA, Ibrahim GH, El Serafi TI, Ghobeish AA (2010) Evaluation of urinary proepithelin as a potential biomarker for bladder cancer detection and prognosis in Egyptian patients. Cancer Biomark 7: 163-170.

44. Donald CD, Laddu A, Chandham P, Lim SD, Cohen C, et al. (2001) Expression of progranulin and the epithelin/granulin precursor acrogranin correlates with neoplastic state in renal epithelium. Anticancer Res 21: 3739-3742.

45. Ho JC, Ip YC, Cheung ST, Lee YT, Chan KF, et al. (2008) Granulin-epithelin precursor as a therapeutic target for hepatocellular carcinoma. Hepatology 47: 1524-1532.

46. Cheung ST, Wong SY, Leung KL, Chen X, So S, et al. (2004) Granulin-epithelin precursor overexpression promotes growth and invasion of hepatocellular carcinoma. Clin Cancer Res 7629-7636.

47. Cheung ST, Wong SY, Lee YT, Fan ST (2006) GEP associates with wild-type p53 in hepatocellular carcinoma. Oncol Rep 15: 1507-1511.

48. Cheung ST, Cheung PF, Cheng CK, Wong NC, Fan ST (2011) Granulinepithelin precursor and ATP-dependent binding cassette (ABC)B5 regulate liver cancer cell chemoresistance. Gastroenterology 140: 344-355.

49. Chen XY, Li JS, Liang QP, He DZ, Zhao J (2008) Expression of PC cell-derived growth factor and vascular endothelial growth factor in esophageal squamous cell carcinoma and their clinicopathologic significance. Chin Med J (Engl) 121: 881-886.

50. Line A, Stengrevics A, Slucka Z, Li G, Jankevics E, et al. (2002) Serological identification and expression analysis of gastric cancer-associated genes. $\mathrm{Br} J$ Cancer 86: 1824-1830

51. Wang H, Sun Y, Liu S, Yu H, Li W, et al. (2011) Upregulation of progranulin by Helicobacter pylori in human gastric epithelial cells via p38MAPK and MEK1/2 signaling pathway: role in epithelial cell proliferation and migration. FEMS Immunol Med Microbiol doi: 10.1111/j.1574-695X.2011.00833.x.[Epub ahead of print]

52. Kong WJ, Zhang SL, Chen X, Zhang S, Wang YJ, et al. (2007) PC cell-derived growth factor overexpression promotes proliferation and survival of laryngeal carcinoma. Anticancer Drugs 18: 29-40.
53. Diamandis EP, Goodglick L, Planque C, Thornquist MD (2011) Pentraxin-3 is a Novel Biomarker of Lung Carcinoma. Clin Cancer Res 2395-2399.

54. Liau LM, Lallone RL, Seitz RS, Buznikov A, Gregg JP, et al. (2000) Identification of a human glioma-associated growth factor gene, granulin, using differentia immuno-absorption. Cancer Res 60: 1353-1360.

55. Martert JM, Fuller CM, Gillespie GY, Bubien JK, McLean LA, et al. (2001) Differential gene expression profiling in human brain tumors. Physiol Genomics 5: 21-33.

56. Kim CH, Cheong JH, Kim JM (2010) Correlation of granulin expression in intracranial meningiomas to clinial parameters. Experimental and Therapeutic Medicine 1: 493-496.

57. Wang W, Hayashi J, Kim WE, Serrero G (2003) PC cell-derived growth factor (granulin precursor) expression and action in human multiple myeloma. Clin Cancer Res 9: 2221-2228.

58. Larramendy ML, Niini T, Elonen E, Nagy B, Ollila J, et al. (2002) Overexpression of translocation-associated fusion genes of FGFRI, MYC, NPMI, and DEK, but absence of the translocations in acute myeloid leukemia. A microarray analysis. Haematologica 87: 569-577.

59. Laha T, Pinlaor P, Mulvenna J, Sripa B, Sripa M, et al. (2007) Gene discovery for the carcinogenic human liver fluke, Opisthorchis viverrini. BMC Genomics 8: 189 .

60. Smout MJ, Laha T, Mulvenna J, Sripa B, Suttiprapa S, et al. (2009) A granulinlike growth factor secreted by the carcinogenic liver fluke, Opisthorchis viverrini, promotes proliferation of host cells. PLoS Pathog 5: e1000611.

61. Miki K (2011) Gastric cancer screening by combined assay for serum antiHelicobacter pylori IgG antibody and serum pepsinogen levels-"ABC method". Proc Jpn Acad Ser B Phys Biol Sci 87: 405-414.

62. Zanocco-Marani T, Bateman A, Romano G, Valentinis B, He ZH, et al. (1999) Biological activities and signaling pathways of the granulin/epithelin precursor Cancer Res 59: 5331-5340.

63. Lu R, Serrero G (2001) Mediation of estrogen mitogenic effect in human breast cancer MCF-7 cells by PC-cell-derived growth factor (PCDGF/granulin precursor). Proc Natl Acad Sci USA 98: 142-147.

64. He Z, Bateman A (2003) Progranulin (granulin-epithelin precursor, PC-cellderived growth factor, acrogranin) mediates tissue repair and tumorigenesis. $J$ Mol Med 81: 600-612.

65. He Z, Ismail A, Krizahev L, Sadvakassova G, Bateman A (2002) Progranulin (PC-cell-derived growth factor/acrogranin) regulates invasion and cell survival. Cancer Res 62: 5590-5596

66. Youn BS, Bang SI, Kloting N, Park JW, Lee N, et al. (2009) Serum progranulin concentrations may be associated with macrophage infiltration into omental adipose tissue. Diabetes 58: 627-636

67. Tangkeangsirisin W, Hayashi J, Serrero G (2004) PC cell-derived growth factor mediates tamoxifen resistance and promotes tumor growth of human breast cancer cells. Cancer Res 64: 1737-1743.

68. Pizarro GO, Zhou XC, Koch A, Gharib M, Raval S, et al. (2007) Prosurvival function of the granulin-epithelin precursor is important in tumor progression and chemoresponse. Int J Cancer 120: 2339-2343.

69. Ismail A, Bateman A (2009) Expression of TBX2 promotes anchorageindependent growth and survival in the p53-negative SW13 adrenocortical carcinoma. Cancer Lett 278: 230-240.

70. Wong AK, Shanahan F, Chen Y, Lian L, Ha P, et al. (2000) BRG1, a component of the SWI-SNF complex, is mutated in multiple human tumor cell lines. Cancer Res 60: 6171-6177.

71. Hahn WC, Weinberg RA (2002) Modelling the molecular circuitry of cancer. Nat Rev Cancer 2: 331-341.

72. Hahn WC, Counter CM, Lundberg AS, Beijersbergen RL, Brooks MW, et al. (1999) Creation of human tumour cells with defined genetic elements. Nature 400: 464-468.

73. Elenbaas B, Spirio L, Koerner F, Fleming MD, Zimonjic DB, et al. (2001) Human breast cancer cells generated by oncogenic transformation of primary mammary epithelial cells. Genes Dev 15: 50-65. 
Citation: Zhang Y, Bateman A (2011) The Glycoprotein Growth Factor Progranulin Promotes Carcinogenesis and has Potential Value in Anti-cancer Therapy. J Carcinogene Mutagene S2:001. doi:10.4172/2157-2518.S2-001

74. Lundberg AS, Randell SH, Stewart SA, Elenbaas B, Hartwell KA, et al. (2002) Immortalization and transformation of primary human airway epithelial cells by gene transfer. Oncogene 21: 4577-4586.

75. Hamad NM, Elconin JH, Karnoub AE, Bai W, Rich JN, et al. (2002) Distinct requirements for Ras oncogenesis in human versus mouse cells. Genes Dev 16: $2045-2057$

76. Kusakari T, Kariya M, Mandai M, Tsuruta Y, Hamid AA, et al. (2003) C-erbB-2 or mutant Ha-ras induced malignant transformation of immortalized human ovarian surface epithelial cells in vitro. Br J Cancer 89: 2293-2298.

77. Hahn WC, Dessain SK, Brooks MW, King JE, Elenbaas B, et al. (2002) Enumeration of the simian virus 40 early region elements necessary for human cell transformation. Mol Cell Biol 22: 2111-2123.

78. Lu R, Serrero G (2000) Inhibition of PC cell-derived growth factor (PCDGF epithelin/granulin precursor) expression by antisense PCDGF CDNA transfection inhibits tumorigenicity of the human breast carcinoma cell line MDA-MB-468. Proc Natl Acad Sci USA 97: 3993-3998.

79. Kamrava M, Simpkins F, Alejandro E, Michener C, Meltzer E, et al. (2005) Lysophosphatidic acid and endothelin-induced proliferation of ovarian cance cell lines is mitigated by neutralization of granulin-epithelin precursor (GEP), a prosurvival factor for ovarian cancer. Oncogene 24: 7084-7093.

80. Park MY, Park YS, Nam JH (2011) RNA interference against granulin-epithelin precursor prevents hepatocellular carcinoma growth: Its application as a therapeutic agent. Int J Oncol 39: 853-861.

81. Zhang H, Serrero G (1998) Inhibition of tumorigenicity of the teratoma PC cell line by transfection with antisense cDNA for PC cell-derived growth facto (PCDGF, epithelin/granulin precursor). Proc Natl Acad Sci USA 95: 1420214207.

82. Orimo A, Weinberg RA (2006) Stromal fibroblasts in cancer: a novel tumorpromoting cell type. Cell Cycle 5: 1597-1601.

83. Daniel R, He Z, Carmichael KP, Halper J, Bateman A (2000) Cellular localization of gene expression for progranulin. J Histochem Cytochem 48: 999-1009.

84. Tangkeangsirisin W, Serrero G (2004) PC cell-derived growth factor (PCDGF/ GP88, progranulin) stimulates migration, invasiveness and VEGF expression in breast cancer cells. Carcinogenesis 25: 1587-1592.

85. McAllister SS, Gifford AM, Greiner AL, Kelleher SP, Saelzler MP, et al. (2008) Systemic endocrine instigation of indolent tumor growth requires osteopontin. Cell 133: 994-1005.

86. Simpson CD, Anyiwe K, Schimmer AD (2008) Anoikis resistance and tumor metastasis. Cancer Lett 272: 177-185.

87. Guerra RR, Kriazhev L, Hernandez-Blazquez FJ, Bateman A (2007) Progranulin is a stress-response factor in fibroblasts subjected to hypoxia and acidosis Growth Factors 25: 280-285.

88. Van Damme $P$, Van Hoecke A, Lambrechts D, Vanacker P, Bogaert E, et al. (2008) Progranulin functions as a neurotrophic factor to regulate neuite outgrowth and enhance neuronal survival. J Cell Biol 181: 37-41.

89. Ryan CL, Baranowski DC, Chitramuthu BP, Malik S, Li Z, et al. (2009) Progranulin is expressed within motor neurons and promotes neuronal cell survival. BMC Neurosci 10: 130

90. Kao AW, Eisenhut RJ, Martens LH, Nakamura A, Huang A, et al. (2011) A neurodegenerative disease mutation that accelerates the clearnce of apoptotic cells. Proc Natl Acad Sci USA 108: 4441-4446.

91. Higgins MJ, Stearns $V$ (2011) Pharmacogenetics of endocrine therapy for breast cancer. Annu Rev Med 62: 281-293.

92. Lilling G, Hacohen H, Nordenberg J, Livnat T, Rotter V, et al. (2000) Differential sensitivity of MCF-7 and LCC2 cells, to multiple growth inhibitory agents: possible relation to high bcl-2/bax ratio? Cancer Lett 161: 27-34.

93. Clarke R, Skaar TC, Bouker KB, Davis N, Lee YR, et al. (2001) Molecular and pharmacological aspects of antiestrogen resistance. J Steroid Biochem Mol Biol 76: 71-84

94. Diel P, Smolnikar K, Michna H (1999) The pure antiestrogen ICI 182780 is more effective in the induction of apoptosis and down regulation of BCL-2 than tamoxifen in MCF-7 cells. Breast Cancer Res Treat 58: 87-97.

95. Abrhale T, Brodie A, Sabnis G, Macedo L, Tian C, et al. (2011) GP88 (PCCell Derived Growth Factor, progranulin) stimulates proliferation and confers letrozole resistance to aromatase overexpressing breast cancer cells. BMC Cancer 11: 231

96. Wang W, Hayashi J, Serrero G (2006) PC cell-derived growth factor confers resistance to dexamethasone and promotes tumorigenesis in human multiple myeloma. Clin Cancer Res 12: 49-56.

97. Solar P, Sytkowski AJ (2011) Differentially expressed genes associated with cisplatin resistance in human ovarian adenocarcinoma cell line A2780. Cance Lett 309: 11-18.

98. Yeo W, Mok TS, Zee B, Leung TW, Lai PB, et al. (2005) A randomized phase III study of doxorubicin versus cisplatin/interferon alpha-2b/doxorubicin/ fluorouracil (PIAF) combination chemotherapy for unresectable hepatocellular carcinoma. J Natl Cancer Inst 97: 1532-1538.

99. Thomas MB, O'Beirne JP, Furuse J, Chan AT, Abou-Alfa G, et al. (2008) Systemic therapy for hepatocellular carcinoma: cytotoxic chemotherapy, targeted therapy and immunotherapy. Ann Surg Oncol 15: 1008-1014.

100. Stewart DJ (2010) Tumor and host factors that may limit efficacy of chemotherapy in non-small cell and small cell lung cancer. Crit Rev Oncol Hematol 75: 173-234

101. Hu Y, Feng FY, Chen SJ, Gao YN, Xiao T, et al. (2006) Correlation between the expression of PCDGF in serum and the chemotherapeutic sensitivity in NSCLC. Zhonghua Zhong Liu Za Zhi 28: 603-605.

102. Hu Y, Lin DM, Cheng SJ, Liu YN, Feng FY (2006) Influences of PC cell-derived growth factor and breast cancer resistance protein on the curative effects of platinum-based chemotherapeutic regimens for advanced non-small cell lung cancer. Zhonghua Yi Xue Za Zhi 86: 2611-2614.

103.Xia X, Serrero G (1998) Identification of cell surface binding sites for PC-cellderived growth factor, PCDGF, (epithelin/granulin precursor) on epithelial cells and fibroblasts. Biochem Biophys Res Commun 245: 539-543.

104. Culouscou JM, Carlton GW, Shoyab M (1993) Biochemical analysis of the epithelin receptor. J Biol Chem 268: 10458-10462.

105. Parnell PG, Carter BJ, Halper J (1995) Identification of a membrane-associated receptor for transforming growth factor type E. J Recept Signal Transduct Res 15: 747-756.

106. Hu F, Padukkavidana T, Væqter CB, Brady OA, Zheng Y, et al. (2010) Sortilinmediated endocytosis determines levels of the frontotemporal dementia protein, progranulin. Neuron 68: 654-667.

107.Zheng Y, Brady OA, Meng PS, Mao Y, Hu F (2011) C-terminus of progranulin interacts with the Beta-propeller region of sortilin to regulate progranulin trafficking. PLoS One 6: e21023.

108. Park B, Buti L, Lee S, Matsuwaki T, Spooner E, et al. (2011) Granulin is a soluble cofactor for toll-like receptor 9 signaling. Immunity 34: 505-513.

109. Gonzalez EM, Mongiat M, Slater SJ, Baffa R, lozzo RV (2003) A nove interaction between perlecan protein core and progranulin: potential effects on tumor growth. J Biol Chem 278: 38113-38116.

110.Xu K, Zhang Y, Ilalov K, Carlson CS, Feng JQ, et al. (2007) Cartilage oligomeric matrix protein associates with granulin-epithelin precursor (GEP) and potentiates GEP-stimulated chondrocyte proliferation. J Biol Chem 282 11347-11355.

111. Gao X, Joselin AP, Wang L, Kar A, Ray P, et al. (2010) Progranulin promotes neurite outgrowth and neuronal differentiation by regulating GSK-3ß. Protein Cell 1: 552-562.

112. Nedachi T, Kawai T, Matsuwaki T, Yamanouchi K, Nishihara M (2011) anulin enhances neural progenitor cell proliferation through glycogen synthase kinase $3 ß$ phosphorylation. Neuroscience 185: 106-115.

113. McLean GW, Carragher NO, Avizienyte E, Evans J, Brunton VG, et al. (2005) The role of focal-adhesion kinase in cancer - a new therapeutic opportunity. Nat Rev Cancer 5: 505-515.

114. Liu Y, Xi L, Liao G, Wang W, Tian X, et al. (2007) Inhibition of PC cell-derived growth factor (PCDGF)/granulin-epithelin precursor (GEP) decreased cell proliferation and invasion through downregulation of cyclin D and CDK4 and inactivation of MMP-2. BMC Cancer 7: 22.

115. Stiles CD, Capone GT, Scher CD, Antoniades HN, Van Wyk JJ, et al. (1979) Dual control of cell growth by somatomedins and platelet-derived growth factor. Proc Natl Acad Sci USA 76: 1279-1283. 
Citation: Zhang Y, Bateman A (2011) The Glycoprotein Growth Factor Progranulin Promotes Carcinogenesis and has Potential Value in Anti-cancer Therapy. J Carcinogene Mutagene S2:001. doi:10.4172/2157-2518.S2-001

116. Sell C, Rubini M, Rubin R, Liu JP, Efstratiadis A, et al. (1993) Simian virus 40 large tumor antigen is unable to transform mouse embryonic fibroblasts lacking type 1 insulin-like growth factor receptor. Proc Natl Acad Sci USA 90: 11217-11221.

117.Xu SQ, Tang D, Chamberlain S, Pronk G, Masiarz FR, et al. (1998) The granulin/epithelin precursor abrogates the requirement for the insulin-like growth factor 1 receptor for growth in vitro. J Biol Chem 273: 20078-20083.

118. Liu JL, Blakesley VA, Gutkind JS, LeRoith D (1997) The constitutively active mutant Galpha13 transforms mouse fibroblast cells deficient in insulin-like growth factor-I receptor. J Biol Chem 272: 29438-29441.

119.Spence SL, Shaffer AL, Staudt LM, Amde S, Manney S, et al. (2006) Transformation of late passage insulin-like growth factor-I receptor null mouse embryo fibroblasts by SV40 T antigen. Cancer Res 66: 4233-4239.

120.Belcastro V, Siciliano V, Gregoretti F, Mithbaokar P, Dharmalingam G, et al. (2011) Transcriptional gene network inference from a massive dataset elucidates transcriptome organization and gene function. Nucleic Acids Res 39: 8677-8688.

121. Hoque M, Young TM, Lee CG, Serrero G, Mathews MB, et al. (2003) The growth factor granulin interacts with cyclin T1 and modulates P-TEFbdependent transcription. Mol Cell Biol 23: 1688-1702.

122. Hoque M, Tian B, Mathews MB, Pe'ery T (2005) Granulin and granulin repeats interact with the Tat.P-TEFb complex and inhibit Tat transactivation. J Biol Chem 280: 13648-13657.

123. Hoque M, Mathews MB, Pe'ery T (2010) Progranulin (granulin/epithelin precursor) and its constituent granulin repeats repress transcription from cellular promoters. J Cell Physiol 223: 224-233.

124. Ong CH, He Z, Kriazhev L, Shan X, Palfree RG, et al. (2006) Regulation of progranulin expression in myeloid cells. Am J Physiol Regul Integr Comp Physiol 291: 1602-1612.

125. Rademakers R, Eriksen JL, Baker M, Robinson T, Ahmed Z, et al. (2008) Common variation in the miR-659 binding-site of GRN is a major risk factor for TDP43-positive frontotemporal dementia. Hum Mol Genet 17: 3631-3642.

126. Jiao J, Herl LD, Farese RV, Gao FB (2010) MicroRNA-29b regulates the expression level of human progranulin, a secreted glycoprotein implicated in frontotemporal dementia. PLoS One 5: e10551.

127. Wang WX, Wilfred BR, Madathil SK, Tang G, Hu Y, et al. (2010) miR-107 regulates granulin/progranulin with implications for traumatic brain injury and neurodegenerative disease. Am J Pathol 177: 334-345.

128. Wang WX, Kyprianou N, Wang X, Nelson PT (2010) Dysregulation of the mitogen granulin in human cancer through the miR-15/107 microRNA gene group. Cancer Res 70: 9137-9142.

129. Polymenidou M, Lagier-Tourenne C, Hutt KR, Huelga SC, Moran J, et al. (2011) Long pre-mRNA depletion and RNA missplicing contribute to neuronal vulnerability from loss of TDP-43. Nat Neurosci 14: 459-468.

130.Lu R, Serrero G (1999) Stimulation of PC cell-derived growth factor (epithelin/ granulin precursor) expression by estradiol in human breast cancer cells. Biochem Biophys Res Commun 256: 204-207.
131. Piscopo P, Rivabene R, Adduci A, Mallozzi C, Malvezzi-Campeggi L, et al. (2010) Hypoxia induces up-regulation of progranulin in neuroblastoma cell lines. Neurochem Int 57: 893-898.

132. Suzuki M, Yonezawa T, Fujioka H, Matuamuro M, Nishihara M (2001) Induction of granulin precursor gene expression by estrogen treatment in neonatal rat hypothalamus. Neurosci Lett 297: 199-202.

133. Serrero G (2003) Autocrine growth factor revisited: PC-cell-derived growth factor (progranulin), a critical player in breast cancer tumorigenesis. Biochem Biophys Res Commun 308: 409-413.

134. Khwaja FW, Svoboda P, Reed M, Pohl J, Pyrzynska B, et al. (2006) Proteomic identification of the wt-p53-regulated tumor cell secretome. Oncogene 25 : 7650-7661.

135. Xu D, Suenaga N, Edelmann MJ, Fridman R, Muschel RJ, et al. (2008) Novel MMP-9 substrates in cancer cells revealed by a label-free quantitative proteomics approach. Mol Cell Proteomics 7: 2215-2228.

136. Butler GS, Dean RA, Tam EM, Overall CM (2008) Pharmacoproteomics of a metalloproteinase hydroxamate inhibitor in breast cancer cells: dynamics of membrane type 1 matrix metalloproteinase-mediated membrane protein shedding. Mol Cell Biol 28: 4896-4914.

137. Bai XH, Wang DW, Kong L, Zhang Y, Luan Y, et al. (2009) ADAMTS-7, a direct target of PTHrP, adversely regulates endochondral bone growth by associating with and inactivating GEP growth factor. Mol Cell Biol 4201-4219.

138. Kessenbrock K, Frohlich L, Sixt M, Lammermann T, Pfister H, et al. (2008) Proteinase 3 and neutrophil elastase enhance inflammation in mice by inactivating antiinflammatory progranulin. J Clin Invest 118: 2438-2447.

139. Simpkins FA, Devoogdt NM, Rasool N, Tchabo NE, Alejandro EU, et al. (2008) The alarm anti-protease, secretory leukocyte protease inhibitor, is a proliferation and survival factor for ovarian cancer cells. Carcinogenesis 29 : 466-472.

140. Devoogdt N, Rasool N, Hoskins E, Simpkins F, Tchabo N, et al. (2009) Overexpression of protease inhibitor-dead secretory leukocyte protease inhibitor causes more aggressive ovarian cancer in vitro and in vivo. Cancer Sci 100: 434-440.

141. Guo F, Lai Y, Tian Q, Lin EA, Kong L, et al. (2010) Granulin-epithelin precursor binds directly to ADAMTS-7 and ADAMTS-12 and inhibits their degradation of cartilage oligomeric matrix protein. Arthritis Rheum 62: 2023-2036.

142. Okura H, Yamashita S, Ohama T, Saga A, Yamamoto-Kakuta A, et al (2010) HDL/apolipoprotein A-I binds to macrophage-derived progranulin and suppresses its conversion into proinflammatory granulins. J Atheroscler Thromb 17: 568-577.

143. Canuel M, Libin Y, Morales CR (2009) The interactomics of sortilin: an ancient lysosomal receptor evolving new functions. Histol Histopathol 24: 481-492.

144. Carrasquillo MM, Nicholson AM, Finch N, Gibbs JR, Baker M, et al. (2010) Genome-wide screen identifies rs646776 near sortilin as a regulator of progranulin levels in human plasma. Am J Hum Genet 87: 890-897.
This article was originally published in a special issue, Growth factors : Carcinogenesis handled by Editor(s). Dr. Jaroslava Halper, The University of Georgia, USA; Dr. Ian Stuart Zagon, The Pennsylvania State University, USA 